\title{
SETS OF INTERPOLATION FOR MULTIPLIERS
}

\author{
BY BENJAMIN B. WELLS, JR.
}

\author{
Communicated by Ray A. Kunze, June 3, 1970
}

Let $T$ denote the circle and $I$ a closed ideal of $L^{1}(T)$ under convolution. Let $F I$ denote the set of sequences of complex numbers which are Fourier transforms of elements of $I$.

$$
\mathcal{F} I=\left\{\left(\xi_{n}\right): \exists f \in I, \hat{f}(n)=\xi_{n}\right\} .
$$

A subset $E$ of the integers is called a set of interpolation for the multipliers of $\mathscr{F} I(=M(\Im I))$ if every bounded complex sequence defined on $E$ is the restriction to $E$ of a multiplier of $F I$. $E$ is called a Sidon set if every bounded complex sequence on $E$ is the restriction to $E$ of the Fourier transform of some measure on $T$. Answering a question of Y. Meyer we show here that every set of interpolation $E \subseteq Z^{+}$for $M\left(\mathfrak{F} H^{1}(T)\right)$ is a Sidon set.

Let $A(T)$ denote the Banach space of all analytic continuous functions on $T$ equipped with the supremum norm. Let $\beta=H^{1}(T) \hat{\otimes} C(T)$ be the Banach space of all elements of $A(T)$ which can be expressed in the form $\sum_{1}^{\infty} f_{k} * g_{k}$ where $f_{k} \in H^{1}(T), g_{k} \in C(T)$ and such that $\sum_{1}^{\infty}\left\|f_{k}\right\|_{1}\left\|g_{k}\right\|_{\infty}<\infty$. The norm $\|\cdot\|_{\beta}$ in $\beta$ is the infimum over all such representations. Meyer [1] has shown that the dual of $\beta$ is precisely $M\left(\Im H^{1}(T)\right)$.

TheOREM 1. $\beta$ is isometrically isomorphic to $A(T)$.

Proof. It is clear that the natural embedding of $\beta$ in $A(T)$ is norm decreasing. Let $P(\theta)=\sum_{1}^{v} a_{k} \exp \left[i n_{k} \theta\right]$ be an arbitrary analytic trigonometric polynomial and write $e^{i M \theta} P(\theta)$ as

$$
\sum_{n=-N}^{N}\left(1-\frac{|n|}{N}\right) \exp [i(n+N) \theta] * \sum_{k=1}^{p} b_{k} \exp \left[i\left(n_{k}+M\right) \theta\right]
$$

where $b_{k}=a_{k}\left\{1-\left|n_{k}+M-N\right| / N\right\}^{-1}$. Choose $M=N-\left[N^{1 / 2}\right]$ and $N$ larger than $n_{\nu}$. It is clear that as $N \rightarrow \infty, b_{k} \rightarrow a_{k}$ for each $k$. Since the polynomial on the left-hand side is just a translate of the usual Fejer kernel, it has $L^{1}$ norm equal to 1 . By the choice of $M$, the sup norm of the polynomial on the right-hand side tends to $\|P(\theta)\|_{\infty}$ as $N \rightarrow \infty$. Hence

$$
\|\exp [i M \theta] P(\theta)\|_{\beta}<\|P(\theta)\|_{\infty}+\epsilon
$$

AMS 1969 subject classifications. Primary 4258; Secondary 4205.

Key words and phrases. Multiplier, Sidon set, $\Lambda(2)$, set of interpolation. 
for $N$ sufficiently large.

It is clear that $\|P(\theta)\|_{\beta} \leqq\|\exp [i M \theta] P(\theta)\|_{\beta}$ for all positive integers $M$. Hence $\|P(\theta)\|_{\beta} \leqq\|P(\theta)\|_{\infty}$. Since the analytic trigonometric polynomials are dense in $\beta$ the theorem follows.

The following answers a question raised in $[1$, p. 554].

Corollary. $E \subset Z^{+}$is a set of interpolation for $M\left(\mathfrak{F} H^{1}(T)\right)$ if and only if $E$ is a Sidon set.

Proof. The only implication of interest is the "only if" one. Thus assume $E$ is a set of interpolation for $M\left(\mathfrak{F} H^{1}(T)\right)$. It is an easy consequence of the definition that $E$ is a set of interpolation for $M\left(\mathfrak{F} H^{1}(T)\right)$ if and only if the elements of $\beta$ whose spectra are contained in $E$ have absolutely convergent Fourier series. Hence there is some constant $c$, depending only on $E$, such that

$$
\sum_{k=1}^{\nu}\left|a_{k}\right| \leqq c\|P(\theta)\|_{\beta}
$$

for all trigonometric polynomials $P(\theta)=\sum_{k=1}^{v} a_{k} \exp \left[i n_{k} \theta\right]$ with spectrum contained in $E$. Since $\|P(\theta)\|_{\infty}=\|P(\theta)\|_{\beta}, E$ is a Sidon set (cf. [3, p. 121]). Q.E.D.

It is of some interest to compare the above notions of interpolation in $M(\mathscr{F} I)$ with the following definition implicit in [1]: $E$ is said to be a set of E-interpolation if every bounded complex sequence on $E$ is the restriction to $E$ of a multiplier of $F I(E)$ where $I(E)$ is the ideal of all $L^{1}$ functions whose spectrum is contained in $E$. The concept of Sidon set is replaced here by that of $\Lambda(2)$ set. Recall that $E$ is a $\Lambda(2)$ set if every $L^{1}$ function whose spectrum is contained in $E$ is in $L^{2}$.

THEOREM 2. $E$ is a set of E-interpolation if and only if it is a $\Lambda$ (2) set.

Proof. The fact that $\Lambda(2)$ sets are sets of $E$-interpolation is an immediate consequence of the Riesz-Fisher theorem.

Conversely if $E$ is a set of $E$-interpolation and $P(\theta)=\sum a_{k} \exp \left[i n_{k} \theta\right]$ is an $E$-polynomial define $g(t, \theta)=\sum a_{k} \varphi_{k}(t) \exp \left[\operatorname{in}_{k} \theta\right]$ where $\varphi_{k}$ is the $k$ th Rademacher function. Then $g_{t}=\hat{s}_{t} * f$ where $s_{t}$ is the convolution operator from $I(E)$ to $L^{1}(T)$ such that $s_{t}\left(\pi_{k}\right)=\varphi_{k}(t)$.

Let $l_{\infty, E}$ denote the quotient space of $l_{\infty}$ by the closed subspace of those sequences vanishing on $E$. Then since $E$ is a set of $E$-interpolation, the natural map $\sigma: M(\mathscr{F} I(E)) \rightarrow l_{\infty, E}$ is onto, and hence has a bounded inverse. Thus $\left\|s_{t}\right\| \leqq c$ where $c$ is independent of $t$, and $\left\|g_{t}\right\|_{1} \leqq c\|f\|_{1}$. The proof now proceeds as in Theorem 3.1 of [2].

Integrate $\left(\sum\left|a_{k}\right|^{2}\right)^{1 / 2} \leqq 2 \int_{0}^{1}|g(t, \theta)| d t$ with respect to $\theta$ over $[-\pi, \pi]$ and use the above inequality. 
REMARK. In direct analogy to the space $\beta, \beta_{E}=I(E) \hat{\otimes} C(T)$ may be formed. It may be of interest to ask for what sets $E$ it is true that whenever $F \subset E, F$ is a set of $M(\mathscr{F} I(E))$ interpolation if and only if it is Sidon. By Theorem 2 this will fail if $E=F$ and $E$ is taken to be a set which is $\Lambda(2)$ but not Sidon.

\section{REFERENCES}

1. Y. Meyer, Endomorphismes des idéaux fermés de $L^{1}(G)$, classes de Hardy et séries de Fourier lacunaires, Ann. Sci. École Norm. Sup. (4) 1 (1968), 499-580. MR 39 $\# 1910$.

2. W. Rudin, Trigonometric series with gaps, J. Math. Mech. 9 (1960), 203-227. MR $22 \# 6972$.

3. - Fourier analysis on groups, Interscience tracts in Pure Appl. Math., no. 12, Interscience, New York, 1962. MR 27 \#2808.

University of Oregon, Eugene, Oregon 97403

Universidad Tecnica del Estado, Santiago, Chile 